\title{
A SIMPLE METHOD OF STUDYING THE GENERATION OF THROMBIN IN RECALCIFIED PLASMA

\author{
APPLICATION IN THE INVESTIGATION OF HAEMOPHILIA
}

\author{
BY \\ W. R. PITNEY AND J. V. DACIE \\ From the Department of Pathology, Postgraduate Medical School of London
}

(RECEIVED FOR PUBLICATION OCTOBER 30, 1952)

\begin{abstract}
The aim of the treatment of serious haemorrhage in haemophilia is to correct as far as possible the defect of the haemostatic mechanism. For this purpose fresh blood, plasma, or plasma fractions are commonly administered intravenously. After such therapy the whole blood coagulation time may be reduced to normal (Alexander and Landwehr, 1948). However, even with a normal coagulation time, bleeding may continue, suggesting that the haemostatic mechanism of the patient is still abnormal. In such cases the prothrombin consumption test will in fact usually demonstrate subnormal prothrombin conversion.
\end{abstract}

There seems to be at the present time no wholly satisfactory simple method which can be used to determine the anti-haemophilic potency of a preparation in vitro before it is administered to a haemophiliac. Some indication may be obtained by adding dilutions of the test substance, be it plasma or plasma fraction, to the haemophilic plasma and recording the calcium time or the prothrombin consumption index of the mixture. Calcium times, however, parallel whole blood coagulation times, and, like the latter, may give normal results when the coagulation mechanism is still deranged. The results of the prothrombin consumption index tend to be irregular. The purpose of this paper is to report experiences with a technique which has been of use in assessing quantitatively (in vitro) the effect on the abnormally slow coagulation of haemophilic plasma of the addition to it of normal plasma or its fractions.

The technique consists in following, by a twostage method, the generation of thrombin when plasma or plasma mixtures are recalcified. The generation of thrombin by haemophilic plasma follows a pattern which usually differs strikingly from the normal, and varying degrees of correction of this abnormality can be demonstrated by adding normal plasma or plasma fractions to the haemophilic plasma. A limited experience of this test performed on patients who had received transfusions of plasma has shown that it is at least as sensitive as the prothrombin consumption index in demonstrating the degree to which the coagulation abnormality has been corrected. It has the added advantage that it gives some insight into the dynamics of thrombin formation.

\section{Methods}

Principle of the Test.-If calcium is added to citrated plasma a fibrin clot develops after a varying interval of time. The time which elapses between the addition of the calcium and the appearance of the clot is the "calcium time." The appearance of a clot is not, however, the end-point of the reactions which are occurring in the mixture. Normally, thrombin continues to be generated in increasing amounts for some time after clotting has occurred. The rate of generation then diminishes and the thrombin concentration drops, due to the action of antithrombin. By transferring samples of the recalcified plasma at regular intervals into tubes containing a standard fibrinogen solution, the concentration of thrombin at any particular moment can be inferred from the speed of clotting of the fibrinogen. The concentration of thrombin is inversely proportional to the time which it takes to clot fibrinogen. By reference to a correlation graph prepared by adding known dilutions of a solution of thrombin to tubes containing the fibrinogen solution, the thrombin concentration in the recalcified plasma at the time of taking any particular subsample can be expressed in " units" of thrombin. In this way a thrombin generation curve can be prepared which illustrates the generation of thrombin and its subsequent decay in plasma which has been recalcified. Such a graphic representation of thrombin levels gives more information than does the calcium time alone, which merely indicates the time at which there is sufficient thrombin to clot fibrinogen. Plasma from patients with thrombocytopenia and from patients on dicoumarol therapy, as well as haemophilic plasma partially corrected by therapeutic plasma infusions, may all give 
abnormal types of thrombin generation curves, although the calcium times may be within the normal range.

The assay of thrombin in recalcified plasma mixtures is no new idea. Sampling from a mixture containing excess thromboplastin into tubes containing fibrinogen is the principle of two-stage prothrombin estimation (Herbert, 1940). A similar principle was employed by Biggs and Macfarlane (1951) in investigating the behaviour of haemophilic plasma to which had been added thromboplastin, and Macfarlane and Biggs (1953) have studied the generation of thrombin in whole blood allowed to clot spontaneously in much the same way.

Collection of Blood.-Venous blood, $4.5 \mathrm{ml}$., was withdrawn from a convenient vein, particular care being taken to avoid contamination with tissue juice and frothing in the syringe. The blood was added to $0.5 \mathrm{ml}$. of $3.8 \%$ sodium citrate solution in a graduated centrifuge tube and carefully mixed by inversion. The citrated blood was then lightly centrifuged at a speed of about 1,000 r.p.m. for two minutes. The effect of this procedure is to obtain a plasma which is still rich in platelets. Centrifugation at higher speeds or for longer times gives a plasma deficient in platelets to a greater or lesser degree, which is itself a potent cause of a slow generation of thrombin (vide infra). The plasma was always tested on the day of collection. Storage for some hours at $4^{\circ} \mathrm{C}$. does not appear to influence the results, but the plasma must on no account be frozen. Frozen plasma, when recalcified after thawing, generates thrombin more rapidly and to higher levels than normal. This is probably due to the destructive action of freezing and thawing upon the platelets.

Fibrinogen.-Preparations of human fibrinogen kindly given us by Dr. A. Kekwick of the Lister Institute have been used. The dried material was reconstituted in distilled water and then diluted in saline to give a final fibrinogen concentration of approximately $100 \mathrm{mg}$. per $100 \mathrm{ml}$.

Procedure.-Volumes, each of $0.1 \mathrm{ml}$., of fibrinogen solution were placed in each of $1275 \times 10 \mathrm{~mm}$. tubes set in a rack in a water-bath at $37^{\circ} \mathrm{C}$. Into another tube were placed $0.25 \mathrm{ml}$. of the plasma to be tested and $0.25 \mathrm{ml}$. of saline, and the mixture allowed to reach the temperature of the water-bath. Then $0.25 \mathrm{ml}$. of previously warmed $\mathrm{M}-40$ aqueous calcium chloride solution was rapidly added. The contents of the tubes were rapidly mixed by inversion and a stop-watch started. Starting at $\mathbf{3 0}$ seconds and then at one-minute intervals, $0.025 \mathrm{ml}$. (or 1 drop) of the reacting mixture was transferred, using a calibrated Pasteur pipette, to successive fibrinogen tubes. The times for the fibrinogen to clot in these tubes were recorded. The determination of the end-point was facilitated by viewing in a good light the image of the tube's content magnified by a concave mirror. The presence of an assistant who could record the clotting times was found to be convenient, although not essential. The fibrinogen clotting times were finally converted from the correlation graph into units of thrombin. At a time which corresponded to the calcium time of the plasma, the reacting mixture itself clotted. This clot was expressed against the wall of the tube with a wooden stick and removed as rapidly as possible. Difficulty was sometimes experienced at this stage with haemophilic plasma, for in haemophilia the fibrin may form in successive waves over a considerable period, so that repeated extractions with the wooden stick may be necessary.

\section{Results}

Thrombin Generation in Normal Plasma.-In Fig. 1 are shown the results obtained with several normal plasmas. The curves illustrated include the maximum and minimum concentrations of thrombin produced by 15 normal plasmas as well as the time necessary to reach these concentrations. After an initial short pause the thrombin concentration in the mixture normally rapidly rises and reaches a peak in from two and a half to four and a half minutes following recalcification. The mixture itself usually clots when the thrombin concentration has reached 1 to 2 units. Thrombin is therefore mostly generated, as is well known, after clotting has taken place. The thrombin concentration subsequently falls off rapidly so that there is very little thrombin detectable at the end of 10 minutes.

Effect of Platelets. - To determine the importance of platelets an experiment was performed in which citrated normal blood was subjected to varying speeds of centrifugation for varying periods of time. Thrombin generation tests were performed on each sample of plasma with the results shown in Fig. 2. The curve with plasma obtained by allowing the red cells to sediment spontaneously and that obtained by centrifuging at a speed of 1,000 r.p.m. for two minutes were almost identical. At greater speeds of centrifugation there was a progressive diminution in the amount of thrombin formed in the mixture, and a lengthening of the time required after recalcification for the reaction to complete itself. Plasmas subjected to 3,000 r.p.m. for five minutes or longer lost their normal capacity for rapidly increasing the thrombin concentration, the curves becoming very similar to those obtained using naturally occurring thrombocytopenic plasmas. Some curves of the latter type are shown in Fig. 3.

These results emphasize the importance of standardizing the degree of centrifugation used in $\propto$ obtaining plasma. In the results discussed in this paper 1,000 r.p.m. for two minutes has been used. 
That centrifugation must be carefully controlled is a criticism of the test. However, in experiments in vivo on haemophiliacs where the curves before and after therapy are to be compared, both preand post-transfusion plasmas can be centrifuged together so as to subject them to the same conditions. Similarly, using the test to compare in vitro the effectiveness of a preparation in correcting the deficiency in a haemophilic plasma, a sample of the same haemophilic plasma is naturally used each time as a substrate.

Thrombin Generation in Haemophilic Plasma. -In Fig. 4 are shown the results obtained with the thrombin generation test on plasmas from eight different patients with haemophilia. Other data obtained at the time these tests were performed are given in Table I.

TABLE I

COAGULATION DATA IN EIGHT CASES OF HAEMOPHILIA

\begin{tabular}{c|c|c|c}
\hline $\begin{array}{c}\text { Curve } \\
\text { No. }\end{array}$ & $\begin{array}{c}\text { Coagulation } \\
\text { Timet } \\
\text { (minutes) }\end{array}$ & $\begin{array}{c}\text { Calcium Time } \\
\text { (seconds) }\end{array}$ & $\begin{array}{c}\text { Prothrombin } \\
\text { Consumption } \\
\text { Index } \\
(\%)\end{array}$ \\
\hline 1 & 20 & 225 & 55 \\
2 & 17 & 375 & 80 \\
3 & $15 \frac{1}{2}$ & 360 & 155 \\
4 & 20 & 375 & $1 \%$ \\
5 & 54 & 570 & 180 \\
6 & 50 & 585 & 130 \\
7 & 100 & 800 & 150 \\
8 & 120 & 960 & 0 \\
\hline
\end{tabular}

* These refer to Fig. 4.

+ The coagulation times were carried out by the Lee-White method at $37^{\circ} \mathrm{C}$.

The pattern of thrombin generation when haemophilic plasma is recalcified, although variable, is distinguishable both from the normal and from that of thrombocytopenic plasmas. After a considerable lag phase the thrombin concentration in the mixture slowly increases and in many cases is only reaching its maximum level at the normal conclusion of the experiment (12 minutes). This gradual evolution is in striking contrast to the rapid increase in thrombin concentration observed in normal plasma. In general, the more severe the grade of the disease as judged by other laboratory criteria, the more abnormal is the shape of the curve. The lowest two curves in this series (Nos. 7 and 8) were from patients with the longest coagulation times; the upper four (Nos. 1 to 4) were from patients with coagulation times of 20 minutes or less.

The Effect of Plasma Transfusion.-Tests were carried out to determine to what extent the abnormal thrombin generation curve of haemophilia could be corrected by the intravenous admin- istration of fresh normal plasma. Plasma was administered within four hours of collection. Venous blood from the patient was withdrawn for testing immediately before the plasma was transfused, and again as soon as the transfusion was completed.

It soon became apparent that it was necessary to transfuse large volumes of fresh plasma in order to. achieve a notable improvement in the thrombin generation curve. The whole blood coagulation time could be brought to within the normal range much more easily. In Fig. 5 is demonstrated the results of a representative trial carried out on a boy aged 14 (Case H). The lower thrombin generation curve in Fig. 5 was obtained with the pre-transfusion plasma ; the upper curve was obtained with plasma withdrawn after $380 \mathrm{ml}$. of fresh (four-hour) normal plasma had been administered over a period of two and a quarter hours; the curve, although improved, was still abnormal. Although the concentration of thrombin generated reached a normal level, there was a considerable delay before the reaction began to accelerate, and the maximum concentration was not reached until six and a half minutes compared with the normal range of two and a half to four and a half minutes. In Table II is shown the effect of the infusion upon other coagulation data. The whole blood coagulation time was reduced to well within the normal range; the calcium time and the prothrombin consumption index were still slightly abnormal.

In Fig. 6 and Table II are illustrated the results of a similar attempt to correct the defective coagulation mechanism in a haemophilic adult (Case D) to whom $600 \mathrm{ml}$. of fresh (four-hour) plasma was administered over a period of 80 minutes. Although the concentration of thrombin generated by the post-infusion plasma was considerably greater than previously, the curve had not returned

TABLE II

EFFECT OF THE INFUSION OF FRESH PLASMA IN TWO HAEMOPHILIACS

\begin{tabular}{|c|c|c|c|c|}
\hline Sample & Case & $\begin{array}{l}\text { Coagu- } \\
\text { lation } \\
\text { Time* } \\
\text { (minutes) }\end{array}$ & $\begin{array}{l}\text { Calcium } \\
\text { Time } \\
\text { (seconds) }\end{array}$ & $\begin{array}{c}\text { Pro- } \\
\text { thrombin } \\
\text { Con- } \\
\text { sumption } \\
\text { Index (\%) }\end{array}$ \\
\hline $\begin{array}{l}\text { Pre-infusion } \\
\text { Post-infusion (after } 3 \dot{80} \\
\text { ml. of fresh normal } \\
\text { plasma) }\end{array}$ & $\mathbf{H}$ & 45 & 450 & 160 \\
\hline $\begin{array}{l}\text { Pre-infusion } \\
\text { Post-infusion (after } 6000 \\
\text { ml. of fresh normal } \\
\text { plasma) } \\
\text { la }\end{array}$ & D & $\begin{array}{l}20 \\
7 \frac{1}{2}\end{array}$ & $\begin{array}{l}375 \\
140\end{array}$ & $\begin{array}{r}155 \\
57\end{array}$ \\
\hline
\end{tabular}

* The coagulation times were carried out by the Lee-White method at $37^{\circ} \mathrm{C}$. 


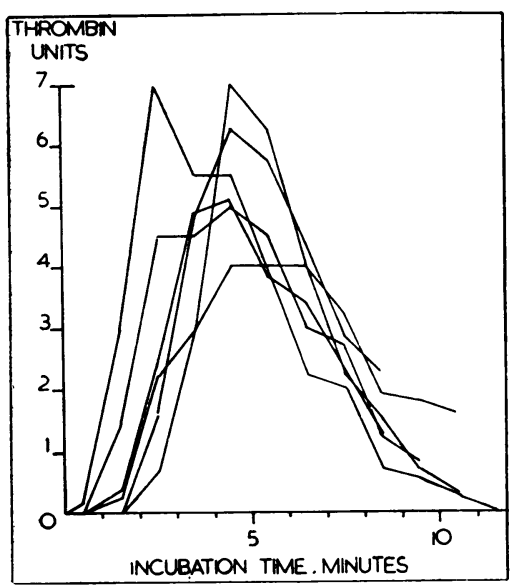

Fig. 1

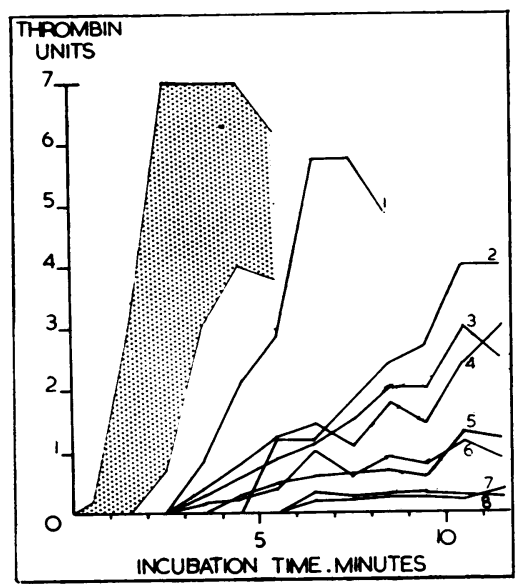

FIG. 4

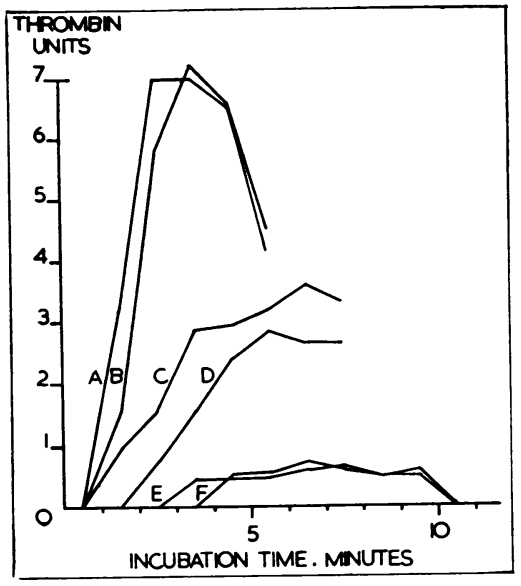

Fig. 2

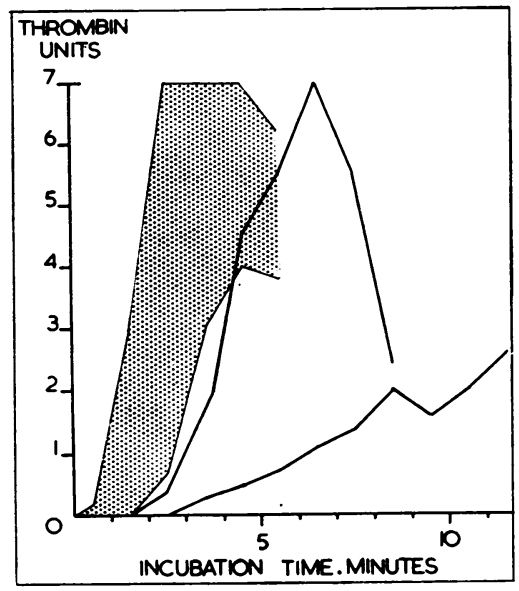

Fig. 5

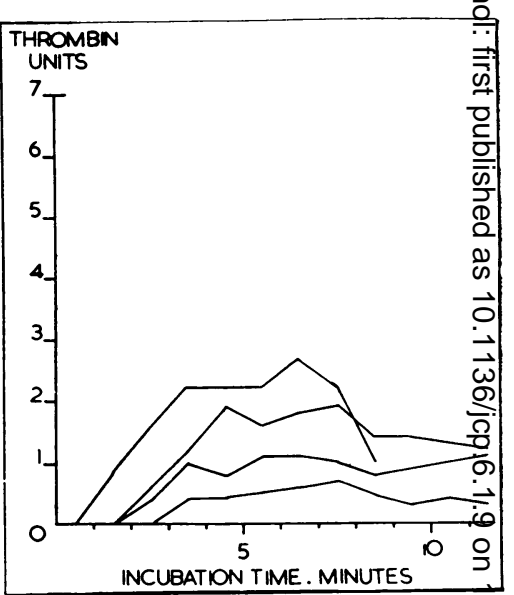

FIG. 3

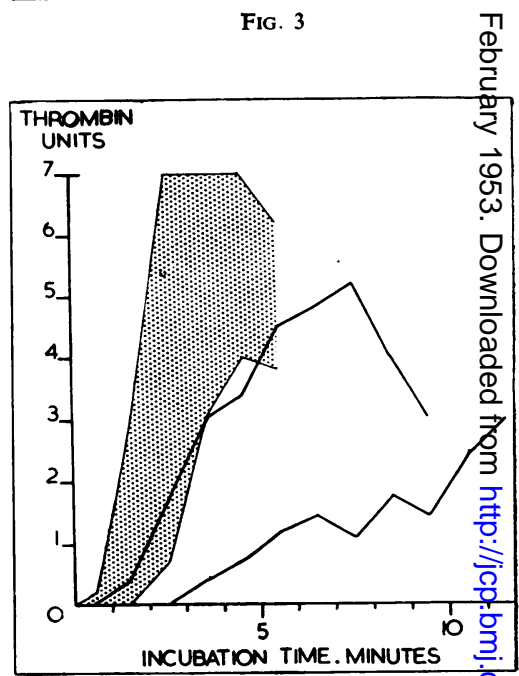

FIG. 6

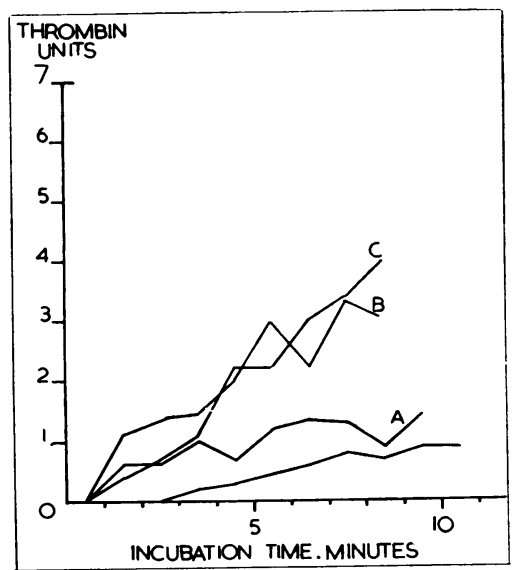

FIG. 7

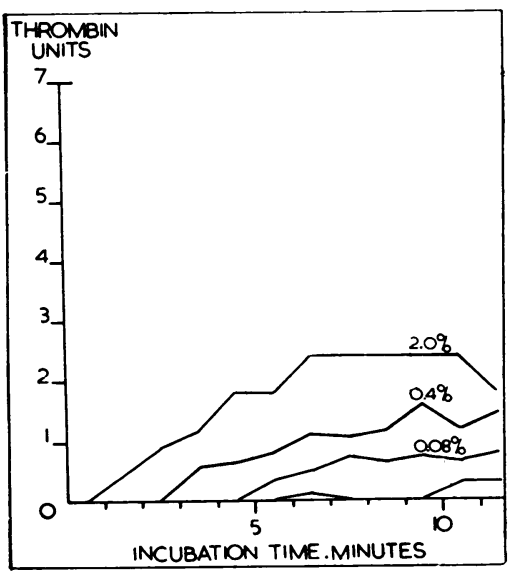

FIg. 8 
to normal. Maximal concentration was not reached until seven and a half minutes. The whole blood coagulation and calcium times were normal ; the prothrombin index was, however, still slightly abnormal.

Experiments in Vitro.-When saline dilutions of normal plasma are added to equal volumes of haemophilic plasma and the mixtures recalcified the pattern of thrombin generation is found to differ from that of the haemophilic plasma alone. Depending on the concentration of the normal plasma added, the thrombin generation curves show varying degrees of partial correction. The addition of as little as $1 \%$ of normal plasma will improve the haemophilic thrombin generation curve, but large amounts have to be added, up to $20 \%$ or even more, to bring the curve to within the normal range. This is in accordance with the results of the in vivo experiments given previously, when $380 \mathrm{ml}$. and $600 \mathrm{ml}$. respectively of fresh plasma were unable to correct the abnormality completely.

FIG. 1.-The generation of thrombin in recalcified normal plasma. The six curves shown include the extremes observed in a range of 15 normal plasmas.

FIG. 2.-Thrombin generation in recalcified normal plasma previously subjected to varying degrees of centrifugation. Curve $A$ : Plasma was obtained by allowing the red cells to sediment spontaneously. Plasma for the other curves was obtained from blood centrifuged as follows: B, 1,000 r.p.m. for two minutes; C, 2,000 r.p.m. for two minutes; D, 2,000 r.p.m. for four minutes; E, 3,000 r.p.m. for five minutes; F, 3,000 r.p.m. for 30 minutes.

FIG. 3.-Thrombin generation in recalcified thrombocytopenic plasmas. These curves were obtained from the plasmas of two patients with idiopathic thrombocytopenic purpura, one patient with aplastic anaemia, and one patient with leukaemia; the platelet counts on whole blood varied from 15,000 to 70,000 per c. mm. The greatest amount of thrombin was generated in the plasma from the patient with the platelet count of 70,000 per c.mm.; the least in the plasma from the patient with the count of 15,000 per c.mm.

FIG. 4.-Thrombin generation in eight recalcified haemophilic plasmas. The range of thrombin generation by normal plasmas is illustrated for comparison.

FIG. 5.-Thrombin generation in a recalcified haemophilic plasma before and after the administration of $380 \mathrm{ml}$. fresh plasma to the patient (a boy, aged 14). The normal range is illustrated for comparison.

FIG. 6.- Thrombin generation in a recalcified haemophilic plasma before and after the administration of $600 \mathrm{ml}$. fresh plasma to the patient (aged 46). The normal range is illustrated for comparison.

FiG. 7.-The comparative ability of fresh and stored plasma in correcting thrombin generation in haemophilia. Curve $A$ was obtained after the addition of $1 \%$ of stored plasma to haemophilic plasma; Curve B after $1 \%$ of freshly frozen plasma; and Curve C after $2 \%$ of stored plasma. The thrombin generation in the haemophilic plasma is illustrated by the bottom line.

Fig. 8. -The effect on the generation of thrombin in a haemophilic plasma of the addition of a fibrinogen fraction of normal plasma. The thrombin generation in the haemophilic plasma is illustrated by the bottom line.
Effect of Storage upon Anti-haemophilic Potency of Normal Plasma.-It is the accepted clinical belief that fresh plasma has greater antihaemophilic potency than has plasma which has been stored for a number of days in a blood bank (Davidson, Epstein, Miller, and Taylor, 1949). On the other hand, the experiments in vitro of Taylor, Lozner, Davidson, Tagnon, and Newhouser (1944) indicated that the anti-haemophilic component of plasma might be preserved intact in unfrozen plasma for as long as six months. That the antihaemophilic factor in normal plasma may deteriorate quite rapidly is illustrated in Fig. 7. Fresh plasma was obtained from a donor and divided into several portions. One sample was immediately placed at $-20^{\circ} \mathrm{C}$. ; the other samples were placed in the blood bank refrigerator at $4^{\circ} \mathrm{C}$. On successive days one sample was removed from the $4^{\circ} \mathrm{C}$. refrigerator and placed at $-20^{\circ} \mathrm{C}$. At the end of 10 days there were thus available in the frozen state samples of the same plasma which had been subject previously to storage at $4^{\circ} \mathrm{C}$. for varying periods of time (0-10 days). The plasma samples were all thawed out together, and their capacity to correct a haemophilic thrombin generation curve compared. Dilutions of the plasmas in saline of $1 \%$ and $2 \%$ were prepared. A volume of these saline dilutions was added to the same volume of haemophilic plasma, the mixtures recalcified, and thrombin generation curves constructed.

The results indicate that about $50 \%$ of the potency of the plasma was lost during storage for 10 days at blood bank temperature. Curves obtained with plasmas stored for intermediate periods of time have been omitted from Fig. 7, but there was in general a progressive diminution in potency paralleling the period of storage. However, the potency of plasma transferred to the freezing cabinet after only one day at $4^{\circ} \mathrm{C}$. did not differ significantly from that of plasma frozen immediately after collection.

The Activity of the Fibrinogen Fraction of Plasma.-It can be demonstrated by fractionation of normal plasma that the anti-haemophilic potency resides mainly in the fibrinogen fraction (Fraction I of Cohn). If prepared from normal plasma, the fraction will accelerate the clotting time of haemophilic blood in vitro and in vivo (Patek and Taylor, 1937; Lozner and Taylor, 1939). Such fibrinogen fractions prepared from normal plasma retain, if freeze-dried, their antihaemophilic potency for long periods of time. It is possible to assess their potency in vitro, using the thrombin generation test. In the experiment illus- 
trated in Fig. $8,0.08 \%, 0.4 \%$, and $2 \%$ dilutions in saline of a fibrinogen fraction were added in equal volumes to haemophilic plasma and the thrombin generated by the mixtures after recalcification estimated. A progressive improvement in the curves was noted with increasing concentration of the fraction. However, even after the addition of $2 \%$ of the fibrinogen fraction the curve was still abnormal. An incorrect impression was given by observations on the calcium time alone. The addition of $2 \%$ of the fraction corrected the calcium time completely ; with $0.4 \%$ of the fraction the calcium time was only just abnormal.

\section{Discussion}

The experiments reported in this paper were chosen as examples to illustrate the usefulness of the thrombin generation test in the investigation of haemophilia. Essentially a simple technique, the test gives valuable information that may not be revealed by other methods of testing. The whole blood coagulation time and the calcium time merely indicate the time necessary, under standard conditions, for the development of sufficient thrombin to clot the plasma fibrinogen. These times may be normal in haemophilia. The prothrombin consumption index indicates the amount of prothrombin remaining in blood a fixed period of time after clotting has taken place. This test is unduly influenced by the presence of accelerators in serum (de Vries, Alexander, and Goldstein, 1949 ; Alexander and de Vries, 1949). The thrombin generation test, by providing a series of points on a curve, allows a more accurate comparison of one plasma sample with another than is provided by either the calcium time or the prothrombin con- sumption index, both of which depend on single observations. The thrombin generation test can be applied to the study of therapeutic problems in haemophilia ; and its use illustrates most strikingly the variability in severity of cases of haemophilia.

\section{Summary}

A simple technique is described for the study of thrombin generation in recalcified citrated plasma, using the method of subsampling into fibrinogen solution. Fibrinogen clotting times are converted into thrombin units and thrombin generation curves constructed.

Examples are given of the use of the technique in the testing of plasma or plasma derivatives for anti-haemophilic potency both in vivo and in vitro. The thrombin generation test is thought to give more information and to be more accurate than the estimation of the calcium time or the prothrombin consumption index.

Experiments are described which give an indication of the relatively iarge volumes of normal plasma or fibrinogen fraction which are needed for the complete correction of the clotting defect in haemophilia.

\section{RFFERENCES}

Alexander, B., and Landwehr. G. (1948), J. Amer. med. Ass. 138 174 .

-

Biggs. R., and Macfarlane, R. G. (1951). Journal of Clinical Patho$\log y, 4,445$

Davidson, C. S., Epstein, R. D., Miller, G. F., and Taylor, F. H. L. (1949). Blood. 4. 97.

Herbert, F. K. (1940). Biochem. J., 34, 1554

Lozner, E. L., and Taylor, F. H. L. (1939), J. clin. Invest . 18. 821

Macfarlane, R. G., and Biggs, R. (1953). Journal of Clinical Patho $\log v, 6,3$

Patek, A. J., and Taylor, F. H. L. (1937). J. clin. Invest , 16, 113

Taylor, F. H. L.. Lozner, E. L., Davidson, C. S. Tagnon. H. J., and Newhouser, L. R. (1944). Ibid 23, 351 .

Vries, A de..Alexander, B.. and Goldstein. R. (1949). Blood, 4. 247 